\title{
From the Case Records of Kanchi Kamakoti CHILDS Trust Hospital: Fever with Vesiculobullous Lesions
}

\author{
Balasubramanian Sundaram ${ }^{1}$, Dhanalakshmi K${ }^{2}$, Sabapathy L Raj ${ }^{3}$, Sumanth Amperayani ${ }^{4}$, Silky Agrawal ${ }^{5}$
}

Pediatric Infectious Disease (2020): 10.5005/jp-journals-10081-1273

\section{Dr S Lakshan RaJ}

Sir, today we would like to discuss a child who presented with fever with vesiculobullous lesions.

A 3-year-old boy has been bought with fever (documented $T_{\max }$ $102^{\circ} \mathrm{F}$ ), loose stools, and vomiting for 5 days. He also developed bullous lesions over both lower limbs since day 1 of illness, which gradually increased in size. He had no trauma preceding the illness nor history of any previous skin lesion or major illness in the past requiring hospitalization except one episode of simple febrile seizure at 11 months of age. His weight and height were less than 3 rd centile for his age. There is history of sibling death at 18 months of age and the exact cause of death is not known (records not available). Parents said that the sibling died of fever and respiratory distress for 2 days and died in a village medical center.

On examination, he was pale and had vesiculobullous lesions predominantly near the dorsum, posterior aspect of leg, elbow, and scrotal region without any surrounding erythema or pus (Fig. 1). He also had small non-blanchable palpable purpuric lesions all over the body, more on extremities. Systemic examination revealed moderate hepatomegaly and tachycardia with wide pulse pressure and cold extremities. His capillary blood sugar value was $41 \mathrm{mg} / \mathrm{dL}$. I have started him on $0.9 \%$ normal saline bolus along with dextrose bolus and started on broad-spectrum antibiotics-ceftriaxone, vancomycin, and clindamycin after sending complete blood count (CBC), peripheral smear (PS), renal function test (RFT), liver function test (LFT), urine routine, blood culture and sensitivity, blood grouping, and cross-matching.

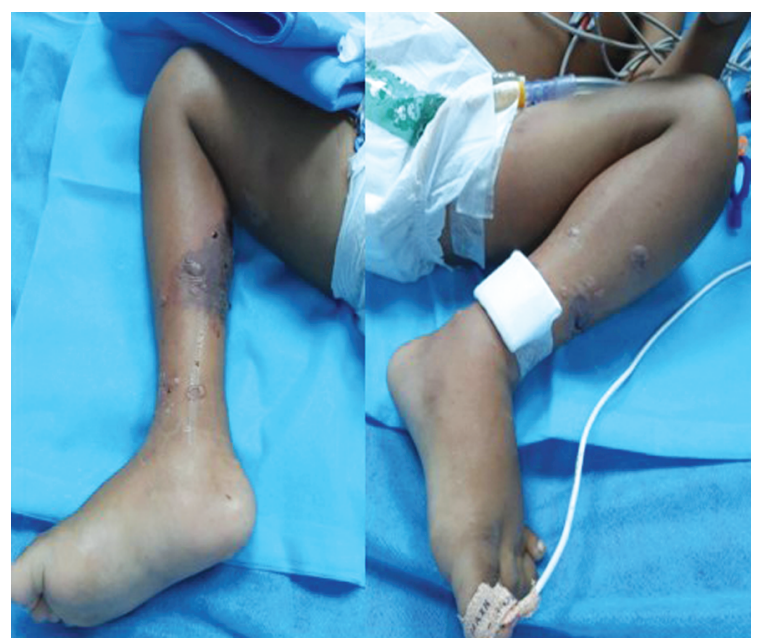

Fig. 1: Vesiculobullous lesions in both lower limbs with edema and areas of necrosis
${ }^{1-5}$ Department of Pediatrics, Kanchi Kamakoti CHILDS Trust Hospital, Chennai, Tamil Nadu, India

Corresponding Author: Dhanalakshmi K, Department of Pediatrics, Kanchi Kamakoti CHILDS Trust Hospital, Chennai, Tamil Nadu, India, Phone: +91 8939345600, e-mail: drkdhana77@gmail.com

How to cite this article: Sundaram B, K Dhanalakshmi, Raj SL, et al. From the Case Records of Kanchi Kamakoti CHILDS Trust Hospital: Fever with Vesiculobullous Lesions. Pediatr Inf Dis 2020;2(3):109-113.

Source of support: Nil

Conflict of interest: None

\section{Dr S Balasubramanian}

This child presented with an acute febrile illness and bullous lesions associated with palpable purpura and he is hemodynamically unstable. This could well be toxic shock syndrome (TSS)/necrotizing fasciitis (NF). The common organism causing TSS is Staphylococcus and group I Streptococcus (GAS) and for NF, apart from Staphylococcus and GAS, gram-negative organisms Pseudomonas aeruginosa, Escherichia coli, Klebsiella, and rarely anaerobes are also implicated. We will escalate the antibiotics to meropenem (from ceftriaxone) and continue vancomycin and clindamycin for antitoxin effect. Dr Silky, could you review literature for causes of fever with bullous lesions in children?

\section{DR SILKY}

Fever and rash implies a broad differential diagnosis. Often the etiology is not obvious and workup involves cultures, skin biopsy, serologies, etc.

\section{Differential Diagnosis for Vesiculobullous Rash and Fever $^{1}$}

- Infectious vesicles:

- Herpes-in single dermatome, pain precedes vesicles; lips, genitals, head and neck of wrestlers, digits of healthcare workers.

- Varicella-different stages of development.

- Variola-same stages of development.

- Rickettsialpox (transmitted by mite bites)_-papule with central vesicle evolves into 1 to $2.5 \mathrm{~cm}$ painless crusted black eschar with erythematous halo and proximal adenopathy.

- Coxsackie A16 vesicles on hands, feet, and mouth of kids.

- Molluscum contagious virus induces flaccid vesicles.

- Infectious bullae:

- Staphylococcal scalded skin syndrome (SSSS) in neonates $\mathrm{d} / \mathrm{t}$ toxin.

- Necrotizing fasciitis and gas gangrene.

() The Author(s). 2020 Open Access This article is distributed under the terms of the Creative Commons Attribution 4.0 International License (https://creativecommons. org/licenses/by-nc/4.0/), which permits unrestricted use, distribution, and non-commercial reproduction in any medium, provided you give appropriate credit to the original author(s) and the source, provide a link to the Creative Commons license, and indicate if changes were made. The Creative Commons Public Domain Dedication waiver (http://creativecommons.org/publicdomain/zero/1.0/) applies to the data made available in this article, unless otherwise stated. 
- Scabies-bullous variant.

- Pseudomonas causing ecthyma gangrenosum in immunocompromised patients with disseminated bullae and sepsis.

- Autoimmune

- Bullous pemphigoid (BP): Blistering skin lesions due to autoantibodies (ABs), typically flexural surfaces, groin, and axillae, occur in middle-aged to elderly. Pruritus is common. It can be bullae or urticarial plaques. IgG and C3 deposits in BM. Drug triggers penicillamine, captopril, and furosemide.

- Pemphigus vulgaris (PV): Starts in oral mucosa, spreads to face, axilla, groin. Mostly flaccid bullae. IgG deposits between epidermal cells.

- Drug reaction, e.g., toxic epidermal necrolysis (TEN)/Steven Johnson syndrome (SJS).

\section{Dr Balasubramanian}

Among these, autoimmune etiology is unlikely because of the age and very short duration of illness and also there is no history of drug intake prior to the onset of illness to think of drug reactions like SJS/TEN. So, we are dealing with probably an infectious cause. Dr Sumanth, why did this child present with unusual severity?

\section{Dr Sumanth}

With the background of failure to thrive and sibling death, I would like to rule out immunodeficiency in this child.

\section{Dr LAKSHAN}

The preliminary reports are available. CBC is suggestive of severe anemia $(7.9 \mathrm{~g} / \mathrm{dL})$, leukopenia $\left(1,800\right.$ cells $\left./ \mathrm{mm}^{3}\right)$, and neutropenia [absolute neutrophil count (ANC) 36]. Platelet counts are normal. C-reactive protein (CRP) is very high (252 $\mathrm{g} / \mathrm{L}$ ). He also has deranged coagulopathy with an international normalized ratio (INR) of 1.9. Peripheral smear is suggestive of microcytic hypochromic anemia with leukopenia. Urinalysis showed proteinuria (1+), RFT, and LFT are normal. Blood culture and urine culture are awaited.

The child had persistent hypotension in spite of two fluid boluses and hence was shifted to pediatric intensive care unit. The child required two inotropes for hemodynamic stability and also was given fresh frozen plasma (FFP) and packed red blood cell (PRBC). How to proceed further Sir?

\section{Dr S Balasubramanian}

With such severe presentation, background of sibling death and the basic labs revealing leukopenia and neutropenia we have to rule out underlying immunodeficiency. So proceed with primary immunodeficiency disorder (PID) workup [human immunodeficiency virus by enzyme linked fluorescence assay (HIV ELFA), nitroblue tetrazolium test (NBT), chest x-ray (CXR), immunoglobulin profile, flow cytometry for lymphocyte subset analysis] and ultrasonogram (USG) abdomen for asplenia.

Since we have a probable diagnosis of TSS, we can consider intravenous immunoglobulin (IVIG) (1 g/kg) after obtaining samples for primary immunodeficiency workup and genetic analysis.

\section{Dr Sumanth}

Sir what is the role of IVIG in TSS?

\section{Dr S Balasubramanian}

The proposed rationale for use of IVIG in TSS is to boost antibody levels via passive immunity in the setting of overwhelming infection. Several other mechanisms have been suggested including opsonization of GAS for phagocytic killing, neutralization of streptococcal toxins, inhibition of inflammatory cytokines, such as tumor necrosis factor-alpha (TNF-alpha) and interleukin-6 (IL-6). ${ }^{2}$

Dr Dhanalakshmi, can you highlight when to suspect immunodeficiency in pediatric population.

\section{Dr Dhanalakshmi}

European Society for Immunodeficiencies (ESID) has listed out 10 warning signs when immunodeficiency has to be strongly suspected but for office practice, it can be easily remembered as follows:

If there is failure to thrive or a history of receiving IV antibiotics and/or significant family history with infections that are:

Severe (requiring intravenous antibiotics and hospital admission).

Persistent (difficult to treat with standard regimens).

Unusual infective organisms (opportunistic pathogens).

Recurrent (repeated infection at the same site or with the same organism).

These infective features have been given the acronym SPUR. ${ }^{3}$ PID should also be suspected in individuals with early onset autoimmune diseases or malignancy.

\section{Dr Balasubramanian}

The microbiologist called now and informed that both the blood cultures (two were sent) are growing P. aeruginosa. Now, the entire clinical presentation looks more like NF rather than TSS. We will stop vancomycin and clindamycin and continue meropenem until sensitivity is available, and Dr Silky, can you briefly discuss about regarding NF?

\section{Dr SiLkY}

Necrotizing fasciitis is a severe and potentially life-threatening soft tissue infection characterized by rapidly progressive necrosis of fascia and subcutaneous tissue along the fascial planes. Necrotizing fasciitis is most commonly seen in lower extremities, followed by abdomen and perineum. ${ }^{4}$

Necrotizing fasciitis may be divided into two microbiological categories: Polymicrobial (type I) and monomicrobial infection (type II):

- Polymicrobial (type I) necrotizing infection is caused by aerobic and anaerobic bacteria. Typically, at least one anaerobic species (most commonly Bacteroides, Clostridium, or Peptostreptococcus) is isolated in combination with Enterobacteriaceae (e.g., Escherichia coli, Enterobacter, Klebsiella, Proteus) and one or more facultative anaerobic streptococci (other than GAS). Obligate aerobes (such as $P$. aeruginosa) are rarely components of such mixed infections.

- Monomicrobial (type II) necrotizing infection is usually caused by GAS or other beta-hemolytic streptococci or Staphylococcus aureus.

Clinical presentation of NF varies from erythema, local warmth, swelling, and tenderness in the infected site in early stage to skin ischemia with blisters and hemorrhagic bullae and gas 
formation leading to crepitus in the overlying skin in advanced infection. ${ }^{5}$ In the fulminant form of NF, patients deteriorate within a few hours, showing extensive necrosis of soft tissue, signs and symptoms of severe septic shock, and multiple organ dysfunction syndrome.

Necrotizing fasciitis is a lethal infection characterized by an insidious clinical presentation and progressive tissue destruction. According to the clinical manifestations of NF, Wang et al. ${ }^{6}$ proposed a staging system based on disease progression:

Early (stage 1) characterized by tenderness to palpitation, erythema and swollen, warm skin.

Intermediate (stage 2) characterized by blisters, bullae, skin fluctuance, and induration.

Late (stage 3) characterized by hemorrhagic bullae, crepitus, and skin necrosis.

Any update on flow cytometry reports and Ig profile and any previous $\mathrm{CBC}$ reports are available for this child?

\section{Dr Sumanth}

Sir, we just got the flow cytometry report:

B lymphocytes $\left(\mathrm{CD} 19^{+}\right)$are totally absent.

Ig profile is also hypogammaglobulinemia (IgG 194; IgA 4; IgM

12; $\lg E$ 63).

CXR is normal, HIV ELFA is negative, and NBT uptake is normal.

On retrieval of old records, he had neutropenia (TLC-4,900 cells/ $\mathrm{mm}^{3}$ with ANC-392) (Fig. 2).

\section{Dr Balasubramanian}

Good, as we suspected this child has an underlying primary immunodeficiency and our timely intervention with escalation of antibiotics (for gram-negative coverage) and IVIG has helped this child to recover from septic shock. With the history of sibling death, male gender, absent CD19 cells, and very low Ig levels, the probability of this being $X$-linked agammaglobulinemia $(X L A)$ is high. We need to do genetic workup for confirmation of diagnosis. Dr Lakshan, can you review the literature whether this child fits with the criteria for XLA?

\section{Dr LaKSHan}

X-linked agammaglobulinemia ${ }^{7}$ is a primary humoral immunodeficiency characterized by severe hypogammaglobulinemia, antibody deficiency, and increased susceptibility to infection. Clinical symptoms (infections) are generally first noted in male infants between 3 and 18 months of age.

$\mathrm{X}$-linked agammaglobulinemia is due to defects in a signal transduction molecule called Bruton tyrosine kinase (Btk). Patients who present because of clinical symptoms are usually initially identified by significant hypogammaglobulinemia/ agammaglobulinemia and the near absence of $\mathrm{CD} 19^{+} \mathrm{B}$ cells. The diagnosis is then confirmed with molecular studies identifying a mutation in the BTK gene. There is only one characteristic physical finding of XLA and that is the absence or near absence of the B cell-rich tonsils and adenoids. Palpable lymphoid tissue is absent in some patients.

It is important to obtain a detailed family history since a substantial minority of patients $(41 \%)$ has had an affected male family member (7) (Table 1).

Management ${ }^{7,8}$ includes mainly:

Replacement of immunoglobulin is the cornerstone of treatment for XLA.

General supportive care includes measures to avoid infection and immunization with killed vaccines. Liveviral vaccines are contraindicated. Immunization against transmissible infectious agents is encouraged for close family members.

\begin{tabular}{|c|c|c|c|c|c|}
\hline Sr. No. & $\begin{array}{l}\text { Lymphocyte } \\
\text { subpopulation }\end{array}$ & $\begin{array}{l}\text { Result \% } \\
\text { lymphocyte }\end{array}$ & $\begin{array}{l}\text { Absolute } \\
\text { lymphocyte } \\
\text { count } / \mathrm{mm}^{3}\end{array}$ & $\begin{array}{l}\text { Reference range for } \\
\text { absolute } \\
\text { count } / \mathrm{mm}^{3}\end{array}$ & $\begin{array}{l}\text { Reference } \\
\text { range for \% }\end{array}$ \\
\hline 1 & Lymphocytes & 98.2 & 2484.40 & $2300-5400$ & \\
\hline 2 & $\begin{array}{c}\text { CD19 }^{+} \\
\text {B lymphocytes }\end{array}$ & 0.0 & 0.0 & $390-1400$ & $14-33$ \\
\hline 3 & $\begin{array}{c}\mathrm{CD}^{+} \\
\text {T lymphocytes }\end{array}$ & 96.4 & 2394.06 & $1400-3700$ & $56-75$ \\
\hline 4 & $\begin{array}{l}\mathrm{CD}^{+} / \mathrm{CD}^{+} \\
\text {Th lymphocytes }\end{array}$ & 71.2 & 1770.13 & $700-2200$ & $28-47$ \\
\hline 5 & $\begin{array}{l}\mathrm{CD}^{+} / \mathrm{CD}^{+} \\
\text {Tc lymphocytes }\end{array}$ & 20.7 & 516.64 & $490-1300$ & $16-30$ \\
\hline 6 & CD4/CD8 ratio & \multicolumn{2}{|c|}{3.4} & \multicolumn{2}{|c|}{$0.68-2.73$} \\
\hline 7 & HLA DR & 1.2 & 31.05 & $160-3700$ & $8-45$ \\
\hline 8 & $\begin{array}{c}\mathrm{CD}^{\circ} / \mathrm{CD} 16^{+} 56^{+} \\
\text {NK Cells }\end{array}$ & 1.8 & 45.17 & $130-720$ & 04-17 \\
\hline
\end{tabular}

Fig. 2: Lymphocyte subset analysis 
Monitoring the patient for subclinical, but progressive, pulmonary damage is also important since a number of studies have shown that these patients can develop chronic pulmonary disease.

\section{DR SILKY}

Sir, after 72 hours of hospitalization, there bullous lesions have become more necrotic and indurated. Sensitivity pattern of Pseudomonas suggests that it is pan sensitive, should we de-escalate the antibiotics or continue with the meropenem? (Fig. 3).

\section{Dr S Balasubramanian}

These lesions looks like ecthyma gangrenosum. Get a surgical consult for the debridement of the lesion. We will deescalate

Table 1: Diagnostic criteria for X-linked agammaglobulinemia ${ }^{7}$

Definitive diagnosis
Male patient with less than $2 \% \mathrm{CD} 19^{+} \mathrm{B}$ cells and at least one of the
following:
(1) Mutation in Btk.
(2) Absent Btk mRNA on northern blot analysis of neutrophils or
monocytes.
(3) Absent Btk protein in monocytes or platelets.
(4) Maternal cousins, uncles or nephews with less than $2 \% \mathrm{CD} 19^{+}$
B cells.

Probable diagnosis

Male patient with less than $2 \% \mathrm{CD}_{19}{ }^{+} \mathrm{B}$ cells in whom all of the following are positive:

(1) Onset of recurrent bacterial infections in the first 5 years of life.

(2) Serum IgG, IgM, and IgA more than 2 SD below normal for age.

(3) Absent isohemagglutinins and/or poor response to vaccines.

(4) Other causes of hypogammaglobulinemia have been excluded.

\section{Possible diagnosis}

Male patient with less than $2 \% \mathrm{CD} 19^{+} \mathrm{B}$ cells in whom all of the following are positive:

(1) Onset of recurrent bacterial infections in the first 5 years of life.

(2) Serum IgG, IgM, and IgA more than 2 SD below normal for age.

(3) Absent isohemagglutinins.

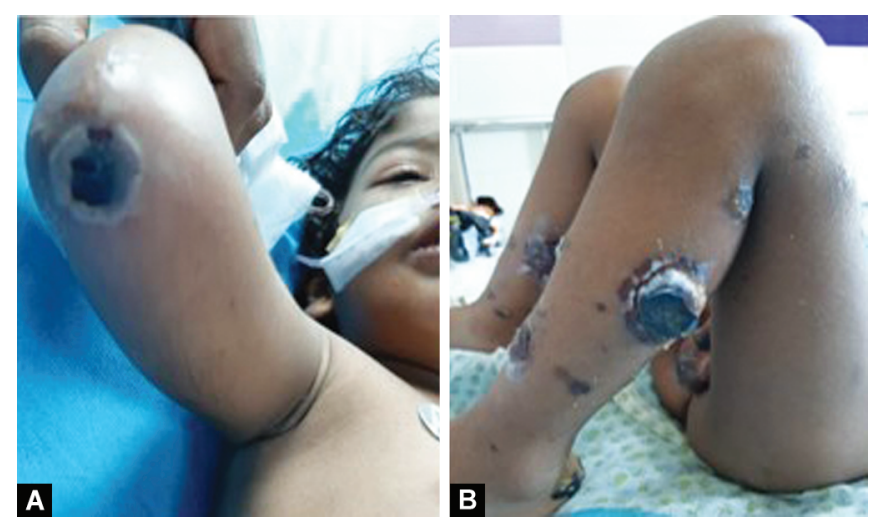

Figs $3 \mathrm{~A}$ and $\mathrm{B}$ : Ecthyma gangrenosum due to Pseudomonas to ceftazidime IV. Dr Silky, is there a need for dual antibiotic in Pseudomonas bacteremia and is there an advantage of adding aminoglycoside and Dr Sumanth, kindly brief on ecthyma gangrenosum?

\section{DR SILKY}

Septicemia and other aggressive infections with Pseudomonas should be treated with either one or two bactericidal agents. Although the number of agents required is controversial, the evidence continues to suggest that the benefit of adding a second agent is questionable, even when studies have included immunosuppressed patients. Whether the use of two agents delays the development of resistance is also controversial, with evidence both for and against. Gentamicin or another aminoglycoside may be used concomitantly for synergistic effect. ${ }^{9}$

Bowers et al., from their study, suggested that there is no difference in mortality outcomes associated with the number of appropriate agents administered during initial empirical therapy for $\mathrm{Pa}$ bacteremia, as long as at least one agent is active. ${ }^{10}$ The addition of an aminoglycoside to an antipseudomonal beta-lactam penicillin does not improve the clinical efficacy achieved with the beta-lactam penicillin alone. ${ }^{11}$

\section{Dr Sumanth}

Pseudomonas aeruginosa generally causes infection in patients with immunodeficiency conditions. Skin lesion characteristic of $P$. aeruginosa septicemia is called ecthyma gangrenosum (EG). ${ }^{12}$ The characteristic clinical appearance is a red maculae that progress to a hemorrhagic bluish bullae that rupture to form a central area of necrosis surrounded by an erythematous halo.

The main site of EG lesions is the gluteal or perineal region, although this lesion can spread to other body sites as occurred in our patient, in which metastatic lesions appeared on both trunk and lower extremities. One major clinical feature in almost all patients is the presence of neutropenia. In two retrospective studies, all the patients with EG were immunocompromised leading to severe neutropenia, and the absolute neutrophil count of less than 500/ $\mathrm{mm}^{3}$ were strongly related to the clinical outcome.

The association of these four components: Sepsis, EG, $P$. aeruginosa, and agammaglobulinemia was first described by Speirs et al. ${ }^{13}$ in 1963 and other authors have also described this rare condition.

Prognosis in P. aeruginosa infection is highly associated with early institution of optimal antibiotic therapy. ${ }^{12-15}$ While awaiting bacteriology results, empirical antimicrobial biotherapy should be started with antipseudomonal beta-lactam penicillin, and adjusted immediately once the bacterial culture results are known.

\section{DR LAKSHAN}

Sir, surgical team has planned to do escharotomy once complete demarcation has occurred. Repeat counts is in the improving trend and fever spike are settling down and child is getting shifted out of intensive care. We have switched over to ciprofloxacin as discussed.

\section{DR SiLKY}

Child has undergone escharotomy and spit skin graft. We have completed a total of 14 days of antibiotics. The wound looks healthy and child is clinically well (Figs 4 and 5). 


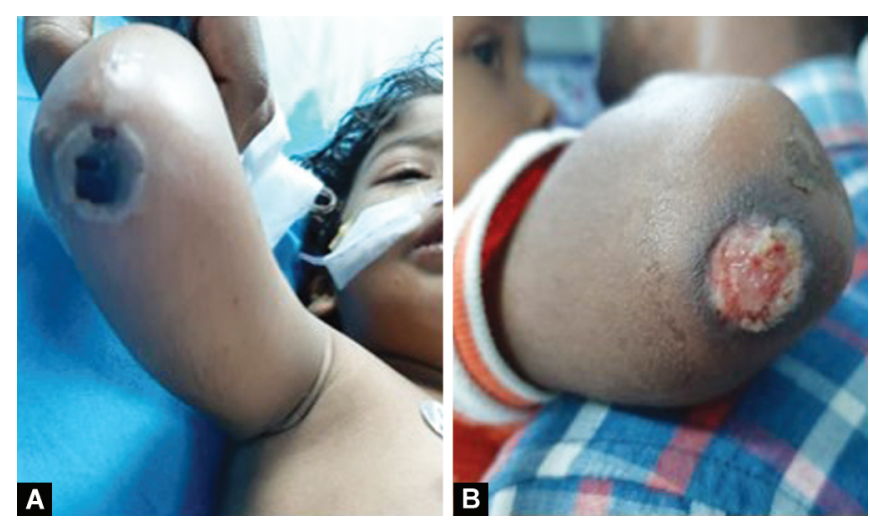

Figs 4A and B: Pre- and post-escharotomy wound in elbow

\section{Dr S Balasubramanian}

He has recovered very well with 14 days of IV antibiotics and his repeat blood culture is negative. We will give oral ciprofloxacin for 2 more weeks and then we will start on oral cotrimoxazole and itraconazole prophylaxis once ciprofloxacin course is completed. I have counseled the parents regarding the need for follow-up and monthly replacement dose of IVIG until we have a confirmed genetic diagnosis. We will send genetic sequencing during follow-up as blood transfusion has been given now.

Learning points from this case:

- In a sick child with vesiculobullous lesions, apart from TSS, other causes like NF should also be considered.

- Judicious and timely use of empirical antibiotics will be lifesaving.

- IVIG should be considered whenever there is a high suspicion of TSS.

- High index of suspicion should be present for immunodeficiency if there is severe, persistent, unusual, and recurrent infections or family history of sibling death is present.

\section{References}

1. http://errolozdalga.com/medicine/pages/KC/RashFever. RashVesiculobullous.4.10.11.html.

2. Waddington CS, Snelling TL, Carapetis JR. Management of invasive group A streptococcal infections. J Infect 2014;69(Suppl 1):S63. DOI: 10.1016/j.jinf.2014.08.005.

3. Primary immunodeficiency diseases 278 CME August 2012 Vol. 30 No.8.

4. Schröder A, Gerin A, Firth GB, et al. A systematic review of necrotising fasciitis in children from its first description in 1930 to 2018. BMC Infect Dis 2019;19(1):317. DOI: 10.1186/s12879-019-3941-3.
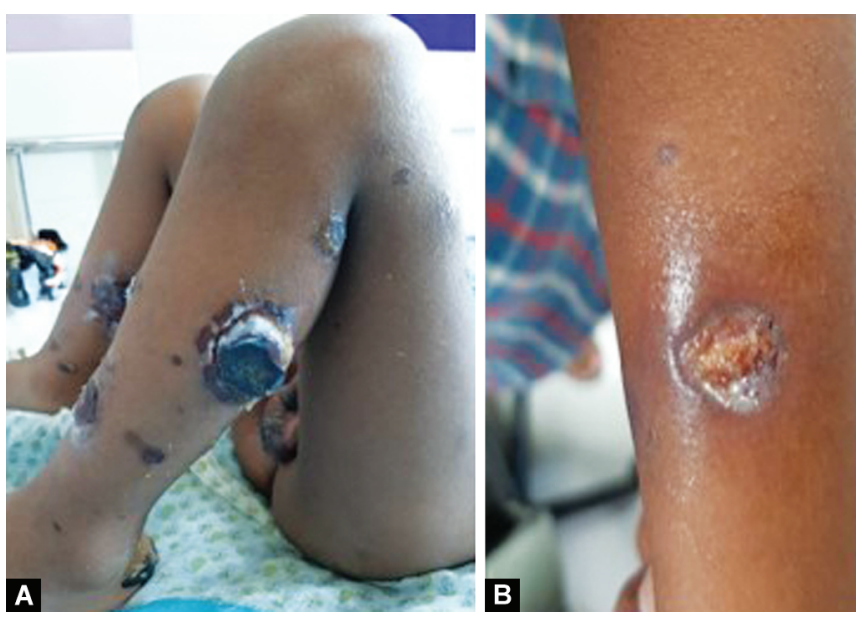

Figs $5 \mathrm{~A}$ and $\mathrm{B}$ : Pre- and post-escharotomy wound in leg

5. Misiakos EP, Bagias G, Patapis $P$, et al. Current concepts in the management of necrotizing fasciitis. Front Surg 2014;1:36. DOI: 10.3389/fsurg.2014.00036.

6. Wang YS, Wong CH, Tay YK. Staging of necrotizing fasciitis based on the evolving cutaneous features. Int J Dermatol 2007;46(10):10361041. DOI: 10.1111/j.1365-4632.2007.03201.x.

7. Conley ME, Rohrer J, Minegishi Y. X-linked agammaglobulinemia. Clin Rev Allergy Immunol 2000;19(2):183. DOI: 10.1385/CRIAI:19:2:183.

8. Ochs HD, Stiehm ER, Winkelstein JA. Antibody deficiencies. Immmunologic disorders in infants and children Stiehm ER, Ochs HD, Winkelstein JA, ed., Philadelphia: Elsevier/Sanders; 2004. p. 356.

9. Nelson - aminoglygoside.

10. Bowers DR, Liew Y-X, Lye DC, et al. Outcomes of appropriate empiric combination versus monotherapy for Pseudomonas aeruginosa bacteremia. Antimicrob Agents Chemother 2013;57(3):1270-1274. DOI: 10.1128/AAC.02235-12.

11. Paul M, Leibovici L. Combination therapy for Pseudomonas aeruginosa bacteremia: Where do we stand? Clin Infect Dis 2013;57(2):217-220. DOI: 10.1093/cid/cit220.

12. Biscaye $S$, Demonchy $D$, Afanetti M, et al. Ecthyma gangrenosum, a skin manifestation of Pseudomonas aeruginosa sepsis in a previously healthy child. Medicine 2017;96(2):e5507. DOI: 10.1097/ MD.0000000000005507.

13. Speirs CF, Selwyn S, Nicholson DN. Hypogammaglobulinaemia presenting as Pseudomonas septicaemia. Lancet 1963;11(7310):710713. DOI: 10.1016/S0140-6736(63)90350-7.

14. de Almeida JFL, Sztajnbok J, Troster EJ. Pseudomonas aeruginosa septic shock associated with ecthyma gangrenosum in an infant with agammaglobulinemia. Rev Inst Med Trop S Paulo 2002;44(3):167-169. DOI: 10.1590/S0036-46652002000300010.

15. Chan YH, Chong CY, Puthucheary J, et al. Ecthyma gangrenosum: a manifestation of Pseudomonas sepsis in three paediatric patients. Singapore Med J 2006;47:1080-1083. 